\title{
Evidencia: conceptos y usos en la evaluación de políticas y programas públicos
}

\author{
Evidence: concepts and their use in evaluating \\ public policies and programs \\ Myriam Irma Cardozo Brum* \\ Departamento de Política y Cultura, Universidad Autónoma \\ Metropolitana Xochimilco, Ciudad de México, México \\ ORCID: http://orcid.org/0000-0002-5790-6749
}

ISSN-OI85-4259; e-ISSN: 2007-9I76

DoI: http://dx.doi.org/IO.28928/ri/90202I/aot3/cardozobrumm

\begin{abstract}
Resumen
El concepto de evidencia, al igual que otros cercanos como verdad, certeza o razón, se utiliza mucho pero se define poco. El objetivo del presente trabajo es aclararlos y proponer nuevos enfoques teóricos alternativos, que resultan poco conocidos y menos discutidos en América Latina, en materia de políticas y programas públicos, especialmente referidos a su evaluación. El método empleado es la exposición y el análisis de documentos históricos, teóricos y empíricos que se presentan según la siguiente estructura expositiva: I) una revisión de su evolución filosófica en Occidente, bajo diferentes corrientes de pensamiento, 2) un análisis del papel actual que se le otorga a la evidencia en materia de políticas públicas en América Latina, y 3) un estudio focalizado en torno de su uso en la evaluación, resaltando su manejo comunicacional. Se concluye que en la actualidad la "evidencia" tiende a identificarse con el producto de modelos estocásticos y pruebas estadísticas, pero en los procesos comunicativos de políticas se ha vuelto un símbolo poderoso de persuasión que caracteriza un discurso fuertemente positivista, acompañado de otro conjunto de términos considerados valiosos con los que se identifica, como objetividad, rigurosidad, cientificidad, robustez, experimentación y estandarización, entre otros.
\end{abstract}

Palabras clave: verdad, certeza, probabilidad, argumentación, comunicación

\begin{abstract}
The concept of evidence, like other similar ones such as truth, certainty, or reason, are widely used, but poorly defined. The objective of this work is to clarify them and to propose new ones from alternative approaches, less known and less analyzed in Latin America. The method used is the exposition and analysis of historical, theoretical and empirical documents, which we present in the following structure: I) a review of its philosophical evolution in the West under different streams of thought, 2) an analysis of the current role of evidence in Latin American public policies, and 3) a study specially focused on the use of evidence in evaluation of public policies, highlighting its communicational use. It is concluded that, at present, the 'evidence' tends to be identified with the product of stochastic models and statistical tests. But in the communicative processes of policies, it has become a powerful symbol of persuasion, which characterizes a strongly positivist discourse. This is accompanied by another set of terms, considered valuable, with which it is identified, such as objectivity, rigor, scientific, robustness, experimentation and standardization, among others.
\end{abstract}

Keywords: truth, certainty, probability, argumentation, communication.

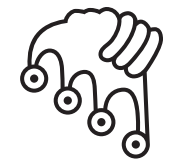

IZTAPALAPA

Agua sobre lajas

* mcardozo@correo.xoc. uam.mx 


\begin{abstract}
¿Dónde reside la verdad? ¿Fuera de nosotros? ¿Dentro de nosotros? ¿En Dios? ¿En las cosas del mundo? ¿En las evidencias más simples o en las teorías más complicadas? ¿En la razón o en el corazón? ¿En la eternidad o en la historia? $¿$ En el individuo o en la colectividad? (Droit 20II: 2I).
\end{abstract}

\title{
Introducción
}

E xiste una amplia variedad de conceptos de evidencia en la evolución filosófica de Occidente, incluso en la actualidad, dependiendo de las corrientes de pensamiento en las que se inserta. Se ha ido pasando de identificarla con la verdad y la certeza indiscutibles a caracterizarla como el resultado de la aplicación de modelos y pruebas estadísticas sólidas. Adicionalmente, en el campo de la comunicación de las políticas públicas se ha ido adoptando un discurso que se refiere muy insistentemente a la "evidencia científica", generalmente del segundo tipo (probabilístico), pero sin aclararlo expresamente, y a veces incluso en un lenguaje que induce a confundirla con el primer sentido (certeza plena), ejerciendo de esa manera una presión cultural que inhibe la emisión de cuestionamientos. Como se verá más adelante, se trata de persuadir respecto de un enunciado tácito (Majone, I992), destacar el carácter persuasivo de la argumentación (Reygadas, 2009), su contenido de poder (Foucault, 1966; Cejudo, 2010), sugerir decisiones basadas en valores, ideologías, intereses, motivaciones y posiciones (Bracho, 20I0), destacar políticas exitosamente comunicadas (OEA, 2OI2) frente a las cuales el desacuerdo es mal visto si se dicen basadas en el conocimiento científico o técnico (Shore, 2010), entre otros.

Conocer y debatir el planteamiento anterior permitiría a los académicos y practicantes de las políticas públicas y su evaluación reflexionar acerca del papel que cumple el discurso sobre las evidencias en nuestro contexto, cuando se le identifica con un enfoque que se autocaracteriza como único de tipo "científico" y/o cuando la ambigüedad en su interpretación se deja librada al interlocutor. Consecuentemente, 
el objetivo de este artículo es profundizar en las conclusiones antes sintetizadas mediante la presentación y discusión de la revisión realizada, con la intención de elucidar conceptos y proponer enfoques alternativos, poco conocidos y menos discutidos en América Latina. Evidentemente, el trabajo no avanzó en forma lineal, pero aquí se presenta con una estructura ordenada para facilitar la comunicación: I) una revisión de su evolución filosófica en Occidente, bajo diferentes corrientes de pensamiento; 2) un análisis del papel actual que se le otorga a la evidencia en materia de políticas públicas en América Latina; y 3) un estudio especialmente focalizado en torno de su evaluación, resaltando su manejo comunicacional. Las conclusiones parciales del primer punto constituyen el marco teórico de análisis para el segundo, y de igual forma, las del segundo permiten abordar el tercero, objetivo final del trabajo, que se concreta en la discusión final y conclusiones aportadas. El método empleado en esta revisión fue la búsqueda y el análisis de documentos históricos, teóricos y empíricos, siguiendo autores reconocidos, que se consideró realizan aportaciones significativas al tema desde la filosofía, el lenguaje, la comunicación, las políticas públicas y su evaluación. En esta última materia se agregó además una revisión en Internet sobre la utilización recurrente y vaga del concepto de evidencia en diversos países latinoamericanos.

\section{Conceptos y enfoques teóricos sobre la evidencia}

Evidencia es un término muy utilizado pero que resulta confuso, poco definido y muy relacionado con otros tantos conceptos tan complicados y discutibles como verdad, certeza, razón, conocimiento, ciencia, prueba, medida, utilidad, racionalidad, entre otros. Ante la dificultad de precisarlo, algunos autores prefieren señalar lo que consideran que no es - mito, creencia, moda, fe -, aunque en esta diferenciación no hay consenso; mientras otros sienten la necesidad de adjetivarlo para darle mayor fuerza, refiriéndose, por ejemplo, a la evidencia rigurosa, contundente o robusta.

Este trabajo presenta inicialmente algunas definiciones de fuentes reconocidas del concepto, analiza brevemente su evolución en el tiempo y sus variaciones en el marco de diferentes enfoques teóricos y lenguas diversas. Para ello se parte de definiciones de diccionario y se sigue el camino marcado por prestigiados autores de diversas disciplinas, pertinentes para desentrañar su devenir filosófico (Xirau, Reygadas y Contreras, Marías, entre otros), las aportaciones de la lógica (Lefevre, Montes de Oca), la epistemología (Feyerabend, Popper), el lenguaje (Chomsky, De Saussure, Foucault, Reygadas) y el vocabulario jurídico (Couture). 
En torno del primer aspecto, conviene señalar su origen epistemológico del latín èvidens, que significa visible o manifiesto. El Diccionario de la Real Academia Española define a la evidencia como "el conjunto disponible de hechos o de información que indican si una creencia o proposición es verdad o válida”, mientras el Diccionario Panhispánico de Dudas la identifica con la certeza clara y manifiesta de la verdad o realidad de algo. La evidencia así entendida puede ser de tipo ontológico (basada en la percepción de objetos), epistemológico (fundamentada en la experiencia o el conocimiento) o incluso intuitivo. Todo esto parece indicar que algo evidente no deja lugar a dudas; en consecuencia, deberíamos tener consenso respecto de su certeza y verdad. Sin embargo, estas definiciones nos remiten a otras, como habíamos anticipado, sin que exista consenso en torno de conceptos hegemónicos homogéneos y entre los que se observará una suerte de circularidad.

En relación con su evolución y enfoques, en la antigüedad, partimos de la aceptación de algún tipo de realidad objetiva, considerada como objeto de estudio desde Tales de Mileto (siglo vi a.C.), con base en una combinación de experiencia, racionalidad e imaginación (Xirau, 20II). La certeza era referida como la posesión perfecta de la verdad eterna, absoluta, definitiva, perfecta, perseguida por la metafísica (Lefevre, 198I). Se contraponía a la opinión y la duda, que podían incluir errores o conocimiento insuficiente (Montes de Oca, 1984). Un siglo después, Heráclito de Efeso (siglo v a.C.) señalaba que para descubrir el sentido profundo de la realidad, más allá de las apariencias, los sentidos corporales eran poco útiles, solo la razón podía captar su racionalidad interna. Ya en el siglo iv a.C., Demócrito manifestaba su escepticismo y subjetivismo al afirmar que "no se conoce nada porque la verdad está en lo profundo" y tampoco confiaba en que el conocimiento sensorial fuera suficiente (Reygadas y Contreras, 2009: 144). Esta actitud filosófica fue sustituida en el Medioevo por la predominancia de creencias religiosas, que se fueron desvaneciendo con el Renacimiento.

Desde el siglo xvir, inicio de la Edad Moderna, se comienza a hablar de ciencia, desarrollada a través de dos corrientes opuestas: racionalismo, a partir de Descartes en Francia, y empirismo, iniciado por Bacon en Inglaterra (Xirau, 20II). Ante la duda sobre la posibilidad del conocimiento objetivo de la realidad se establecen exigencias metodológicas estrictas para alcanzar la verdad, ya sea en el plano del razonamiento lógico o de la verificación empírica.

En el caso del racionalismo, la certeza se obtiene privilegiando el método de pensamiento deductivo; implica "la adhesión intelectual a la verdad sin temor a equivocarse", pero también se acepta la intuición, que permite captar una idea "clara 
y distinta" (Montes de Oca, I984: IOI, I37), que no deja duda. En consecuencia, el Discurso del método establece cuatro reglas en que, junto con el análisis, la síntesis y la enumeración, se menciona la evidencia como criterio de verdad. En el empirismo se parte de la observación sensorial de casos de la realidad y mediante el método inductivo se reflexiona hasta alcanzar un conocimiento cuya verdad se verifica mediante contrastación empírica; su evidencia se obtiene especialmente a través de los sentidos. En síntesis, en este periodo se tiene evidencia de fundamento empírico, por un lado, y por otro, basada en la intuición y la lógica formal.

A partir del siglo xix surgen nuevas ideas en el marco de la llamada filosofía contemporánea. Aumenta el número de pensadores que no cree posible alcanzar conocimientos absolutos, crecen la incertidumbre y la ambigüedad, y se diversifican las corrientes de pensamiento (idealismo, marxismo, existencialismo, positivismo, pragmatismo, etc.) que proponen nuevos métodos como la lógica dialéctica, mientras el empirismo se reduce. Para Marías, la realidad es concebida como mundos múltiples, las teorías son diversas, contradictorias y dinámicas: "Ningún sistema puede pretender una validez absoluta y exclusiva, porque ninguno agota la realidad; en la medida en que cada uno de ellos se afirma como único, es falso. Cada sistema filosófico aprehende una porción de la realidad" (Marías, 1980: 5). Frente a la idea desarrollada por el cartesianismo y el idealismo, que consideraba la verdad como coherencia interna del pensamiento, William James retoma un concepto realista: correspondencia entre la conciencia y la cosa. Afirma: "Son ideas verdaderas aquellas que podemos asimilar, validar, corroborar y demostrar; falsas ideas, las que no." (Xirau, 2011: 402).

Por otra parte, el desarrollo científico positivista ha inducido a pensar en una realidad reducida a información cuantitativa, manejable con métodos lógico-matemáticos y una evidencia de hechos percibidos por los sentidos. Pero dicha evidencia también tiende a confundirse con creencias basadas en el comportamiento cultural y ético esperado en la regularidad de la experiencia y su extrapolación al futuro, que no pueden considerarse ciertas, sino meramente probables. Algunas creencias son claramente falsas, pero otras presentan diferentes niveles de racionalidad; todas pueden llegar a ser socialmente admitidas como verdades. Pueden expresarse como mito, religión, arte o ideología, que contribuyen a la identidad del grupo social. Olivé (1988) afirma que tampoco el concepto de racionalidad es claro, está cargado de prejuicios y mitos, pero cumple un importante papel ideológico que se relaciona con la cientificidad, la objetividad y la intersubjetividad.

También han surgido importantes investigaciones que relacionan esos conceptos con el lenguaje, que hace posible su comunicación. El estudio de la lingüística 
estructural (de Saussure, 2008) y la gramática generativa (Chomsky, 1992) permiten comprender la relación entre creencias, evidencias y verdades. Ellas pueden ser tratadas como conjeturas (Popper, 2007), sometidas a procesos de "falsación", y acabar siendo abandonadas.

Por otra parte, el positivismo ha ido enfrentando fuertes cuestionamientos desde el relativismo, la mecánica cuántica, la similitud entre ciencia y mito (Feyerabend, 1975), el teorema de la imposibilidad de Godel, entre otros, limitando la modelización de sistemas consistentes y completos. Lefevre afirma: "Nuestra época está lejos de ser exclusivamente científica. Sigue penetrada por elementos de pensamiento teológicos y metafísicos, [reconoce que] la disyunción racional y rigurosa es un procedimiento indiscutible, pero su alcance es limitado y su campo de aplicación, restringido. [...] Lo real, el contenido, presenta innumerables e imprevisibles matices". (Lefevre, 1981: 82, 105, 107).

La crítica posmoderna a la modernidad va dirigida a su fundamentación en la razón, sus valores y logros materiales alcanzados, así como al tipo de hombre y sociedad que postula. Rechaza la idea de acumulación y progreso del conocimiento, cuestiona las creencias sobre la verdad, el conocimiento, el poder, el individualismo y el lenguaje (Flax, 1987). La investigación científica ya no persigue verdades inmutables, leyes válidas para todo tiempo y lugar, sino verdades útiles (pragmatismo) para el "progreso" social. Esa utilidad se traduce en la obtención de ganancias para el mundo empresarial o en la eficacia para alcanzar objetivos gubernamentales ligados al poder (Lyotard, 1984), que tratan de mantener un mundo unívoco fundado en valores únicos y supuestamente compartidos. Pero la verdad y el método para obtenerla dejan de ser únicos; la verdad se construye (Bachelard, 2000), dando lugar a un nuevo relativismo: el constructivismo. Ningún conocimiento, supuesto, método o teoría se considera superior a otro y la "evidencia conclusiva" no existe. Foucault (1966) señala que la verdad siempre es relativa, legitima el poder en turno y es controlada por este; puede incluso llegar a limitar la manera de pensar (Giddens, 2006).

Lo importante no son ya los hechos sino sus diversas interpretaciones posibles, siempre difusas e inciertas, que provocan diferentes significados en un contexto de relativismo cultural y ético y de secularización de las instituciones modernas. Existen entonces distintos niveles de evidencia, incluso en el conocimiento científico. En este contexto, el lenguaje, estudiado por Heidegger, Gadamer o Derrida, se vuelve central, se considera que todo es texto y que el hombre está inmerso en él.

En torno de la multiplicidad de enfoques teóricos, los principales (racionalismo, empirismo, positivismo, relativismo, pragmatismo) han ido siendo sintéticamente referidos; sin embargo, apenas hemos hecho alusión al constructivismo, que en las 
últimas décadas ha venido ganando fuerza en contextos como Alemania, Francia e incluso los Estados Unidos, y que tiene particular importancia para el caso que en el siguiente apartado nos proponemos analizar.

El constructivismo es una corriente que se generó hace aproximadamente un siglo, impulsada por teóricos opuesto al positivismo como Vygotsky y Mead, Moscovici y Bachelard. Logró salir airosa aunque algunos positivistas prestigiados como Carnap dudaron de la posibilidad de utilizar el método experimental en campos alejados de la física, especialmente en las ciencias sociales, debido a los riesgos éticos, las dificultades metodológicas de experimentar en la sociedad o la posibilidad de que los resultados fueran contraproducentes. No obstante, el constructivismo desapareció hasta retomar su presencia en los años setenta con los trabajos de epistemología genética de Piaget, que plantearon problemas como la separación positivista de sujeto y objeto, reflexión y acción, teoría y práctica, investigación básica y aplicada, conceptos abstractos y experiencias concretas, etcétera.

Le Moigne (1994) vuelve a cuestionarse si la autonomía de la ciencia es posible o se trata de una suerte de religión revelada que hay que respetar. Propone sustituir el reduccionismo racionalista y positivista por el pensamiento complejo (Morin, 1998), sin mutilaciones, captando especialmente las interrelaciones de la realidad. También se retoman posturas críticas como las de Feyerabend o Popper que piensan que la certeza surge más de la convicción personal que de un conocimiento absoluto. La complejidad, la incertidumbre y la imprevisibilidad de fenómenos emergentes se vuelven características intrínsecas de los sistemas sociales. En consecuencia, Le Moigne postula que los objetos de investigación no son dados, sino que "se construyen" de manera interdisciplinaria y con amplios contenidos de participación e interpretación social.

En los Estados Unidos, Berger y Luckmann (1966) sostienen que todo el conocimiento de la realidad diaria se deriva y es mantenido por las interacciones sociales cuyos significados acaban convirtiéndose en facticidades externas e independientes al hombre. Gergen propone que se remplace el supuesto de la verdad verificada mediante la naturaleza, por el de verdad creada (construida) en comunidad."Hablar, entonces, de 'mundo material' y relaciones causales' no es describir de modo exacto lo que existe, sino participar de un género textual: beber de un inmenso repositorio de inteligibilidades que constituyen una tradición cultural particular" (Gergen, 2007: 99). La "objetividad" surge entonces de la intersubjetividad, de la negociación de significados entre la gente. El lenguaje, entonces, vuelve a aparecer como elemento fundamental para trasmitir un mundo difuso, carente de sentido único, y se retoma a De Saussure (2008), quien señala su carácter reificador en las interacciones humanas, 
es decir, que constructos arbitrarios terminan por ser considerados como objetos reales, ajenos a su construcción social. Significante y significado no son estables, se transforman continuamente, provocando que el lenguaje sea relativo y cambiante.

Esto planteamientos también enfrentan críticas desde la relación entre conocimiento y poder (Foucault, 1966). En realidad, los debates epistemológicos se desarrollan junto a los discursos políticos del poder y el control. En fin, para el construccionismo nada es absoluto, universal o eterno.

Antes de dar por terminada esta revisión resulta importante hacer referencia a la evidencia en otro campo de investigación (policial y criminalística) y en otro contexto geográfico. Dentro del primero, los antecedentes datan por lo menos de la mitad del siglo XIX (Bentham, I843). Actualmente se distinguen tres conceptos claves y cercanos: indicio, prueba y evidencia (Couture, 2005). La evidencia se entiende como todo elemento que permite establecer de manera clara la relación entre otros dos elementos encontrados en la escena del delito (ej. huellas dactilares). En este sentido, la evidencia se usa para probar, en lugar de que se presenten pruebas que conduzcan a producirla, como señala la Academia; indica "si una creencia o proposición es verdad o válida”.

Por último, dos palabras sobre el uso de "evidencia" en el idioma inglés. Evidence es toda prueba que se alega en un proceso judicial; y agrega que, en español, solo sería aceptable como sinónimo de prueba evidente, esto es, prueba clara y manifiesta. En esta definición, entonces, evidencia es sinónimo de prueba; nuevamente, no se trata de un conjunto de pruebas que conducen a la evidencia (en singular), sino que puede haber evidencias diversas y en sentidos incluso opuestos.

Como síntesis de esta revisión, consideramos que evidencia, verdad y certeza han perdido completamente su sentido original y absoluto, que solo permanecen presentes en los enfoques positivistas aún vigentes y en el lenguaje policial.

\section{Debate teórico sobre el papel de la evidencia en las políticas públicas}

En el caso de las políticas públicas y, como veremos después en su evaluación, el papel que juega el concepto de evidencia es de suma importancia. Al respecto se expone el trabajo de uno de los pocos autores de amplio reconocimiento en este campo de aplicación (Majone), que cuestiona la supuesta precisión y las bases científicas postuladas por el positivismo, destacando el carácter persuasivo, no neutral, de la 
evidencia. Este es seguido de los aportes de autores más recientes que argumentan en forma similar (Cejudo, Bracho, Reygadas y Shore, entre los principales).

Para Majone (1992: 356) la evidencia constituye un aspecto descuidado en el análisis de políticas a pesar de que requiere grandes cantidades de datos, aunque de dudosa confiabilidad y significación. La evidencia no es un dato ni de una información, es "información seleccionada e introducida en un punto específico del argumento para persuadir a un auditorio particular de la verdad o falsedad de un enunciado tácito". Debe valorarse en cada caso en función de muchos factores, incluyendo la capacidad persuasiva del analista.

Un problema importante que Majone encara es el de los modelos diseñados para formular políticas, tratando de emular el éxito de las ciencias "duras". Generalmente, se brindan los resultados de un modelo cuyos supuestos y funcionamiento desconocemos y asumimos sus productos como "hechos", ya que no podemos verificarlos. Modelos diferentes, alimentados con los mismos datos, conducen obviamente a distintas recomendaciones de política. Por eso, deberían constituir solo un elemento más a utilizar en un argumento, junto con otros datos e información. Sin embargo, incluso para que los resultados de los modelos sean usados como evidencia deben satisfacer ciertos requisitos de procedimiento en función de distintos tipos de ella, testimonios, pruebas de hecho, presunciones e interpretaciones, es decir, diferenciar los datos, la información, la evidencia y las conclusiones. Sin embargo, las inferencias realizadas a partir de los datos no suelen someterse a ningún tipo de escrutinio, con lo cual se transforman en actos de fe, como sucede en las religiones, lo que nos recuerda la posición de Feyerabend respecto de la ciencia.

Agrega Majone que el analista difiere mucho del científico, supuestamente neutral según la tradición positivista; el analista es un productor de argumentos para persuadir al decisor en favor de una política. Los cálculos de una evaluación y los instrumentos matemáticos, estadísticos y econométricos que permiten obtenerlos son importantes, pero deben complementarse con una adecuada argumentación que los respalde y los justifique. Esto nos lleva a la necesidad de mejorar los métodos y las condiciones del discurso en todos los niveles y etapas, valiéndonos de la retórica para formular argumentos convincentes e implantar políticas adecuadas, luego de analizar su factibilidad. Con este planteamiento reaparece la importancia del lenguaje en torno de las evidencias.

En similar sentido que Majone, Cejudo menciona que el proceso de políticas públicas, lejos de ser una simple decisión racional, está moldeado "por las instituciones, el entorno organizacional, el marco legal, las condiciones políticas y económicas, y los valores sociales en juego. A esta lista agrega uno más: el discurso" (Cejudo, 2oro: 
93). Propone su estudio desde el enfoque constructivista, para recuperar los significados construidos socialmente, resaltando su carácter persuasivo y su contenido de poder. Es decir que quienes toman decisiones se basan más en narrativas causales que en evidencias. En relación con la evaluación, retoma la cita "los resultados empíricos nunca hablan por sí solos. La valoración de evidencia siempre depende de los criterios usados, y esto criterios están, inevitablemente, determinados por la política" (Fischer, 2003: 9).

Por otro lado, Bracho plantea que su "pregunta central se refiere a las posibilidades de utilizar evidencia en el diseño y la implementación de políticas públicas" (Bracho, 2010: 293). Retoma el trabajo de Simons (2004), quien señala que las políticas ya han sido clasificadas en función del uso que hacen de la evidencia: a) políticas que no toman en cuenta la evidencia (evidence-ignorant policy); b) políticas que están al tanto de la existencia de evidencia pero no la aplican (evidence-aware policy); c) políticas que consideran la evidencia, pero que no están completamente modeladas por ellas (evidence-related policy); d) políticas que se perciben con influencia de la evidencia (evidence-influenced policy); y e) políticas moldeadas y basadas en evidencia intencionalmente buscada, relativa a relaciones causales entre los propósitos buscados, las opciones de acción y los resultados (evidence-based policy). Las últimas dos las considera similares al modelo de solución de problemas (Weiss, 1979).

Bracho también retoma tres de los métodos de generación de evidencia (Davies, 2004): revisiones sistemáticas, estudios referidos a proyectos específicos y estudios piloto y de caso. Estos brindan datos e información que se incorpora a la argumentación o construcción teórica que sustenta su comunicación, como ha señalado Majone. De esta forma, Bracho sintetiza que las Evidence-based Policy o Políticas basadas en evidencia ( $\mathrm{PBE}$ ) son "un tipo de política pública basada en investigación, que aplica procedimientos rigurosos y sistemáticos para la recolección de datos y se preocupa por la transformación de estos en conocimiento formal de carácter utilizable para la toma de decisiones". Finalmente, señala la PвE como "un instrumento de negociación y cabildeo que permita informar a los tomadores de decisión de sus efectos, resultados e impactos, haciéndola más sensible a las necesidades de sus usuarios" (Bracho, 2010ः 307, 31I). Esta autora no ignora las diferencias entre la actividad política y la científica, que dificultan la utilización de la evidencia; tampoco que se requiere una diversidad de enfoques y miradas, o que las decisiones se toman sobre valores, ideologías, intereses, motivaciones y posiciones, pero propone que se utilice la mejor información o evidencia de que se disponga. 
Reygadas también afirma que "Su aporte es decisivo con respecto al debate político, la negociación, la construcción de consensos ciudadanos y la comprensión de una de las operaciones fundamentales que construyen nuestra conciencia por medio del lenguaje, la emoción y la intuición: argumentar" (Reygadas, 2009: 9). Por esto, nos propone estudiar el paso de los simples esquemas lógicos y dialécticos al discurso destinado a persuadir a los demás, en el marco de su contexto, mediante un enfoque interdisciplinario, de complejidad, desde el constructivismo, y focalizado en la intersubjetividad.

Finalmente, Cris Shore (2010) y María Clemencia Ramírez (2010), aunque no se refieren específicamente a la evidencia, incluyen para completar, desde la perspectiva crítica, la visión insinuada por Majone respecto de la formulación de políticas públicas y su relación con el lenguaje. Ramírez señala que las políticas públicas han sido tratadas y percibidas por la sociedad como algo neutro, ajeno a la política $y$ el poder. De ahí la importancia de estudiar las ideologías, los intereses y objetivos concretos de los tomadores de decisiones, así como los significados culturales y símbolos sociales, que Shore presenta como similares al mito en sociedades no letradas, así como postula su necesario estudio interdisciplinario. Este se refiere a la fuerte presencia del positivismo y las teorías de elección racional en los estudios de políticas públicas y su tratamiento como una actividad casi científica, a pesar de la importancia que el lenguaje, la retórica y la persuasión han ido logrando. Considera que las políticas buscan unir a la gente en un fin común y levantar fronteras simbólicas que los diferencien de los demás, a través del lenguaje, discurso y poder, así como del uso de técnicas literarias y teatrales. Resalta que el desacuerdo es mal visto cuando los asuntos se presentan como conocimiento científico o técnico, que debe ser manejado por expertos. Opina que las políticas son "ambiguas y polisémicas, tienen múltiples significados que no pueden ser siempre especificados con precisión científica" (Shore, 2010: 34).

Como hemos podido ver, las políticas públicas pueden nutrirse de "evidencias" como datos, información, pruebas; pero para ser efectivas requieren un lenguaje adecuado, que comunique y persuada de su conveniencia.

\section{Usos de la evidencia en la evaluación de políticas y programas públicos}

La evaluación busca contribuir a mejorar el diseño y la implementaciónde las políticas y los programas públicos para la obtención de mejores resultados e impactos 
en torno de los problemas públicos que encaran. $\mathrm{Al}$ ser parte del proceso seguido por las políticas y los programas, recogen e incluso exacerban la discusión del punto anterior en torno del denominado enfoque de $\mathrm{PBE}$, cuya difusión mundial es importante y casi única en Latinoamérica.

Las conclusiones de las evaluaciones nunca son evidentes en el sentido de ofrecer información cierta, verdadera y suficiente que pueda expresarse en un juicio por todos compartido. Sus conclusiones no son categóricas, muestran algunos aspectos positivos y otros negativos. Estos últimos justifican el trabajo evaluativo al permitir identificar problemas y proponer soluciones que lleven a mejorar el objeto evaluado. Pero también suelen discutirse en función del enfoque teórico asumido (principalmente positivista o constructivista) o los métodos y técnicas aplicados.

Sin embargo, existe una tendencia a hablar de sus resultados como "evidencias", que se presenta a continuación mediante consultas en Internet acompañadas de la revisión literaria pertinente al tema, con la intención de mostrar su extensa difusión, pero sin pretender de ninguna manera ser exhaustivos respecto de su prolífera producción. Por ejemplo, en 2005, la V Conferencia Bienal de la European Evaluation Society (ees), organizada en Sevilla, España, se dedicó a Learning, Theory and Evidence. Se trataba de las tres bases de la evaluación contemporánea (aprendizaje, teorías y pruebas), discusión que no estaba presente en nuestro contexto latinoamericano. Como pude verse, evidence fue traducido como pruebas y referido a las corrientes que buscaban darle un enfoque "científico" al proceso de políticas públicas, trabajando con base en evidencias logradas en pruebas experimentales rigurosas y con normas estrictas (Campbell y Stanley, 2005). En América Latina, evidence aparece emparentada con el positivismo predominante en el desarrollo de evaluaciones, con avances importantes en Colombia, México y Chile, donde prácticamente no se hace mención de las alternativas teóricas presentes en Europa, principalmente el constructivismo.

Las diferencias relevantes entre la tendencia positivista latinoamericana y los contados ejemplos alternativos de evaluación (por ej. en el gobierno de la Ciudad de México), se sintetizan en el cuadro I.

\section{Cuadro i:}

Enfoques de la evaluación: principales características

\begin{tabular}{ll}
\hline Énfasis positivista & Énfasis constructivista \\
\hline $\begin{array}{l}\text { Trata de descubrir una verdad única y obje- } \\
\text { tiva. }\end{array}$ & $\begin{array}{l}\text { Considera que existen múltiples y subjeti- } \\
\text { vas verdades. }\end{array}$ \\
\hline
\end{tabular}




\begin{tabular}{|c|c|}
\hline Énfasis positivista & Énfasis constructivista \\
\hline $\begin{array}{l}\text { Utiliza un método "científico", similar al de } \\
\text { las ciencias físico-naturales. }\end{array}$ & $\begin{array}{l}\text { Emplea métodos diversos de las ciencias } \\
\text { sociales. }\end{array}$ \\
\hline $\begin{array}{l}\text { Considera que todos los programas deben } \\
\text { evaluarse de la misma forma (estandariza- } \\
\text { ción). }\end{array}$ & $\begin{array}{l}\text { Propone que cada evaluación debe cons- } \\
\text { truirse en función de las características } \\
\text { específicas de su objeto. }\end{array}$ \\
\hline $\begin{array}{l}\text { Reconoce los estudios experimentales como } \\
\text { el método más riguroso de medición de eva- } \\
\text { luaciones de impacto. }\end{array}$ & $\begin{array}{l}\text { Utiliza métodos diversos de medición y } \\
\text { explicación del impacto. }\end{array}$ \\
\hline $\begin{array}{l}\text { Valora la cuantificación (medidas y prue- } \\
\text { bas) muy por encima de la información } \\
\text { cualitativa. }\end{array}$ & $\begin{array}{l}\text { Reconoce la contribución tanto de los } \\
\text { métodos cuantitativos como de los cuali- } \\
\text { tativos. }\end{array}$ \\
\hline $\begin{array}{l}\text { Promueve la realización de trabajos por } \\
\text { expertos, reduciendo el papel de la parti- } \\
\text { cipación social. }\end{array}$ & $\begin{array}{l}\text { Realiza trabajos especializados, pero pro- } \\
\text { mueve la participación social. }\end{array}$ \\
\hline $\begin{array}{l}\text { Prioriza el análisis disciplinario, de corte } \\
\text { económico. }\end{array}$ & $\begin{array}{l}\text { Enfatiza la necesidad de contar con equipos } \\
\text { interdisciplinarios, integrados en función } \\
\text { de las necesidades del objeto a evaluar. }\end{array}$ \\
\hline
\end{tabular}

Fuente: Adaptado de Cardozo (20II: 56).

Después de la experiencia mencionada han proliferado las ponencias en Congresos de Ees y artículos publicados en Evidence E Policy Journal, referidos al tema de evidences entendidas como pruebas, que pueden consultarse en la página web de dicha sociedad. En algunos casos se especifica que por evidence se hace referencia básicamente a la documentación del programa, bases de datos y estadísticas oficiales. En general, tratan temas como su medición, su uso y los factores que la afectan, utilizando evidence reviews (documentación tradicional de la intervención), encuestas, entrevistas a informantes-clave, estudios de caso, etc., en campos tan variados como salud, energía, agua potable, cambio climático, entre otros. Algunos trabajos críticos señalan el descuido de la persuasión política y la negociación para incidir en la utilización de evidencias y recomiendan realizar análisis del discurso; otros, la influencia de factores pragmáticos ajenos a la evidencia científica, pero de mayor peso en la toma de decisiones (otros valores e intereses, simplicidad, congruencia con otros programas en curso, etc.), provocando que esta solo cumpla una función simbólica.

El tema de la evidencia se ha vuelto tan relevante que ha dado lugar a la corriente llamada PBE, ampliamente expandida, como veremos a continuación. Se trata de una 
política (Libro Blanco del Gobierno Británico, 1999), impulsada por el entonces primer ministro del Reino Unido, Tony Blair, con base en el antecedente de la "medicina basada en evidencia", que busca evitar el peso de la ideología y las presiones políticas, tomando decisiones en forma objetiva, rigurosa y efectiva, con base en la convicción de que los resultados son más efectivos cuando los programas se diseñan tomando como base la evidencia científica. O sea, se refiere al impacto o la relación causal entre un programa y sus efectos, medidos a través de experimentación. Autores como Cairney (2016) abordan el tema en el contexto británico.

El enfoque ha sido retomado en la Organización de Estados Americanos (OEA). En un trabajo de 2012 se reconoce la ambigüedad del término evidencia, que se entiende como información recolectada a través de un proceso sistemático. En cuanto a la política basada en evidencia sistemática, la ozA considera que es la surgida de investigaciones objetivas, completa y relevante, además de exitosamente comunicada, no solo datos empíricos. Los decisores políticos deben valorar la evidencia útil con base en criterios como relevancia, objetividad, precisión, credibilidad y aplicabilidad, y tomar en cuenta su calidad y costo (Segura, 2012).

Además, la PBE ha tomado especial importancia en Australia, Nueva Zelanda y euA (paladines de la Nueva Gerencia Pública, con la que aparece vinculada), así como en Canadá. Algunas iniciativas de estos países como la Cochrane Collaboration, la Campbell Collaboration, la International Initiative for Impact Evaluation (3ie), el Abdul Jameel Latif Poverty Action Lab (J-PAL LAC), la Alliance for Useful Evidence, la Commission on Evidence-Based Policymaking, y múltiples publicaciones (Coe, 2004; Pawson, 2006; Banks, 2009; Head, 2010; Davies, 2012; French, 2018; Leuz, 20I8; entre muchos otros) muestran su importancia. Incluso en EUA existen páginas que pretenden calificar programas con criterios científicos (la Coalition for Evidence-based Policy entre 2008 y 2015, y la Social Programmes That Work, actualmente) para que gestores públicos identifiquen fácilmente los programas que logran presentar evidencia de nivel superior (Top tier evidence). También es posible encontrar trabajos en este sentido en Alemania (Adam et al., 2018).

También hay críticos en estos países; Parkhurst (20I7), por ejemplo, señala que la mayoría de los trabajos de políticas basadas en evidencia han fallado a la hora de comprender la naturaleza política de la toma de decisiones y su influencia en la forma en que la evidencia puede ser usada.

En España, la entonces Agencia Estatal de Evaluación de las Políticas Públicas y la Calidad de los Servicios (Aeval) definía la evaluación como "un proceso integral $[\ldots]$ que permita alcanzar un juicio valorativo, basado en evidencias" (Aeval, 2oro: I3). Raya y Zalakain (20II) reiteran que no es lo mismo información que evidencia, 
y manifiestan que en España se investiga poco, hay limitada evidencia científica y ella se sustituye con opinión, intuición, inercia, imitación o ideología. La PBE se aplica desde la década de 1980; se usan revisiones sistemáticas y experimentación, de escasa utilidad y alto costo, pero no se incluye la pluralidad de métodos de las ciencias sociales. Citan a Pinilla (2006), quien aporta que los procedimientos científicos y democráticos discurren por cauces separados y tienen objetivos distintos, pero pueden conciliarse en la $\mathrm{PBE}$, que sustituye la búsqueda de políticas óptimas por políticas robustas, definidas como técnicamente correctas y políticamente aceptables.

Pasando a América Latina, una rápida mirada en Internet nos muestra que esta insistencia en la evidencia es compartida por otros países como Colombia, Perú, Ecuador, Chile, Argentina o México. En el primero de ellos, el lema del Sistema de Seguimiento y Evaluación (Sinergia) es "Sinergia, incidiendo con evidencia". Por ejemplo, Jaramillo, López y Castro (2018), responsables de la evaluación de impacto de un programa, expresan que no existe evidencia suficiente para respaldar su efectividad.

En el caso peruano se menciona que una evaluación auténtica, se basa "en evidencias observables y medibles" (Huerta, 20I8: 159). Por su parte, Almeida (2017) presenta trabajos de la Semana de la Evidencia 2017 en Ecuador, denominada “Cómo crear políticas públicas basadas en evidencia empírica?", que promovió el uso de datos y evidencia científica, entendida como información comprobada o comprobable, para la construcción de políticas públicas.

En cambio, la Dirección de Presupuestos de Chile, encargada de las evaluaciones, solo parece referir un curso llamado Incubadora para la Generación de Evidencia en Programas Públicos, en coordinación con la Oficina para Latinoamérica del J-PAL LAC; sin embargo, hay documentos en Internet, supuestamente relacionados con el tema, que no permiten el acceso. Torres (2015) señala la creciente importancia de la evidencia desde la última década del siglo pasado, para remplazar las políticas ideológicamente sustentadas por políticas basadas en la toma racional de decisiones, rigurosa y sistemática. Sin embargo, considera que, a pesar de la idea de certeza asociada con el concepto de "evidencia científica", esta no está exenta de incertidumbres y controversias.

Para ejemplificar el caso argentino nos referimos a un trabajo de Bertranou (2019), que trata de las fallas en la producción de evidencia sobre los problemas públicos y las posibles respuestas de política pública, así como dificultades a la hora de incorporar esta evidencia en el núcleo mismo del proceso decisorio.

En México, el discurso de la evidencia ha sido ampliamente desarrollado, especialmente en referencia a la evaluación de impacto, desde el Consejo Nacional de 
Evaluación de la Política de Desarrollo Social (Coneval) a nivel federal y asumido sin mayor discusión por la mayoría de los órganos estatales y de los profesionales de la evaluación. No hay que olvidar que el lema del Coneval es "lo que se mide se puede mejorar", ${ }^{1}$ reduciendo la evaluación a la medición. Con objeto de mostrarlo, transcribimos párrafos seleccionados de algunos documentos incluidos en su página web (www.coneval.org.mx):

En las últimas décadas, contar con evidencia que sustente la implementación de políticas públicas ha cobrado mayor importancia para la administración pública. Esto significa medir las relaciones causales entre los bienes y servicios que entregan los programas públicos y las problemáticas que pretenden resolver. (Evidencia sobre la efectividad de las políticas sociales: I).

En un país que busca consolidar la democracia y la rendición de cuentas es fundamental que exista evidencia objetiva ... Sería ingenuo pensar que las decisiones presupuestarias sólo se basan en evidencias técnicas... pero sería una falta de respeto a la ciudadanía que las cuestiones políticas siguieran siendo el único criterio para decidir el destino de los recursos de la población (Memorias del Coneval 2006-2015: 13).

El Coneval es una institución que se ha vuelto un referente a nivel nacional e internacional en la generación de información y evidencia objetiva con rigor técnico .... En ese sentido, el Coneval Itiene como propósito fomentar el uso de la evidencia que genera para la toma de decisiones en materia de desarrollo social. (Identificación y clasificación del uso de la información del Coneval para la mejora de la política de desarrollo social: 4).

La evaluación de impacto busca encontrar evidencia sobre el nivel de efectividad de las políticas públicas... (Memorias del Coneval 2006-2016ः 47).

El Informe de Evaluación de la Política de Desarrollo Social 2018 analiza el desempeño de la política de desarrollo social en México con el propósito de que las administraciones (federal, estatales y municipales) cuenten con evidencia e información para la elaboración, monitoreo o evaluación de sus planes y programas de gobierno. Para elaborar este Informe, el Coneval utiliza la evidencia acumulada a lo largo de

1 La expresión original de Peter Drucker tenía un sentido diferente: "lo que no se mide no se puede mejorar". 
diez años... El propósito principal de este informe es mostrar la evidencia sobre la situación actual del país ... (Evaluación de la política de desarrollo social 20I8)

Con la finalidad de proporcionar evidencia útil que contribuya a la integración del Presupuesto de Egresos de la Federación 2020 ... (Información Coneval para el proceso presupuestario: 2020).

El Coneval considera prioritario generar evaluaciones que proporcionen evidencia sobre los resultados de los programas sociales respecto a las problemáticas que atienden.... que contribuyan a disponer de evidencia sobre la efectividad de los programas sociales... (Incubadora de evaluaciones de impacto, s/f)

A estos documentos pueden agregarse participaciones del entonces secretario ejecutivo del Coneval, como un capítulo de libro y una nota que presentan la política de nutrición basada en la evidencia (Hernández y Mujica, 2008). Pero el tema de la evidencia aparece también en eventos académicos, cursos y manifestaciones de organizaciones patronales y civiles:

- Seminario "io años del Coneval: pobreza multidimensional y buenas prácticas de monitoreo y evaluación 2015". Una de las cinco mesas organizadas se dedicó a "Uso de la evidencia para el diseño de la política pública", con dos ponencias provenientes del Banco de México y la Suprema Corte de Justicia de la Nación que plantean la necesidad de contar con evidencia científica y empírica en sus actividades.

- La “Semana de la Evaluación 20rg” que incluyó los Paneles “Uso, generación de evidencia y aprendizaje participativo","Uso de evidencia para la toma de decisiones en las entidades federativas" $y$ "Monitoreo y evaluación con el uso de evidencia en la política pública", así como la Mesa redonda "Evaluación y democracia: el papel de la evidencia en la toma de decisiones".

- "La evaluación en la política basada en evidencia". Se encuentra en un video sobre evidencias en la evaluación del desempeño de programas públicos, que forma parte del Diplomado de Evaluación de Políticas y Programas Públicos, impartido por la Unidad de Evaluación del Desempeño de la Secretaría de Hacienda y Crédito Público.

- La Confederación Patronal de la República Mexicana (2019), señala que "México necesita datos confiables de los tres órdenes de gobierno para poder desarrollar política pública con base en evidencia." 
- Gestión Social y Cooperación, AC (Gesoc) se presenta como un "organismo de la sociedad civil especializado en la generación de evidencia y soluciones metodológicas útiles y robustas para que las iniciativas sociales de los gobiernos..."

Varios trabajos mexicanos mencionan las metodologías propuestas por el enfoque, como la revisión sistemática (Flores-Crespo, 2013), los estudios experimentales o cuasiexperimentales de impacto y estadísticas de Big Data o grandes masas de datos sobre procesos y productos para el monitoreo periódico (Davies, 2004). Flores-Crespo reconoce que la evidencia no se logra solo privilegiando técnicas cuantitativas.

En un estudio realizado por el Centro para el Aprendizaje sobre Evaluación y Resultados para América Latina y el Caribe (Clear), se muestra que existe un bajo nivel de utilización de la evidencia en varios países latinoamericanos como Colombia, Costa Rica o Perú, entre otros (Pérez y Maldonado, 2015), problema no exclusivo de América Latina en referencia a resultados de evaluación (Cardozo, 2015). Este sólo alcanza un nivel intermedio en Brasil, Chile y México. La evidencia, calificada como sólida o rigurosa se refiere a los resultados o productos de las evaluaciones efectuadas. El Centro para el Aprendizaje en Evaluación y Resultados para América Latina y el Caribe (Clear) presenta muchos motivos por los que la PBE no avanza, como costos monetarios, tiempo y otros factores políticos, sociales y económicos.

\section{Discusión}

En nuestra cultura actual, términos como evidencia, certeza o verdad aún conservan parte de la connotación absoluta que se les atribuyó en la Antigüedad. La evaluación de políticas y programas públicos y su retroalimentación para mejorar el diseño de estos ha tomado frente a ella dos actitudes distintas.

Por un lado, quienes, desde la aproximación positivista, tratan de convencer de que solo su modelo de gestión se apega a esas ideas absolutas y, en consecuencia, debe aplicarse ignorando toda alternativa, como se ha mostrado en la revisión realizada, especialmente en América Latina. En la mayoría de los casos no se define la evidencia, pero se hace referencia a diferentes aspectos complementarios entre sí y vinculados con ella: documentación, bases de datos, estadísticas oficiales y demás información recolectada en forma sistemática, resultados de pruebas rigurosas o investigaciones objetivas completas y relevantes. La "evidencia" aparece como un 
efecto casi mágico que emana de los datos y métodos revisados para aportar si no certeza, al menos convicción. Lo mismo sucede con las PBE en América Latina, en que la "evidencia científica" se dice utilizada como insumo de una toma de decisiones racional y rigurosa. En el caso mexicano, Coneval no la define, pero la identifica con datos confiables y robustos, tratamiento técnico riguroso y medición de impacto experimental o cuasiexperimental.

Por otra parte, quienes defienden una actitud crítica y constructivista de evaluación, casi inexistente en los gobiernos de América Latina y con poca presencia en sus trabajos académicos, hemos visto que se apegan al relativismo filosófico que en ciencias sociales fue impulsado por los trabajos de Kuhn, Lakatos y Feyerabend, así como por el posmodernismo. A estos trabajos se agregan los estudios sobre el papel del lenguaje, la argumentación o el discurso del poder, retomados en las políticas públicas. En consecuencia, su concepto de "evidencia" se aproxima a un argumento basado en información estadística seleccionada y poder, que moviliza valores y emociones, con objetivo de persuadir a otros, en un contexto legal, institucional, económico y social particular. En otras palabras, la evidencia podría considerarse el efecto conjunto de datos, pruebas, argumentos y emociones que coinciden en señalar un desempeño similar de una política o programa, dentro de un discurso $y$ en un contexto de poder.

Lamentablemente, en este momento, la pandemia del Covid-I9 muestra políticas nacionales de salud diferentes, aunque todas argumentan basarse en evidencia científica, con posiciones enfrentadas sobre uso de cubrebocas, diagnósticos, posibles tratamientos, importancia relativa de la salud y la economía, entre otros. México, a pesar de su exitosa comunicación pública diaria, basada en datos y argumentación para persuadir sobre el enfoque adoptado, no deja de enfrentar dudas. Considero que se trata de un excelente ejemplo de la ausencia de evidencia, justificada por la reciente aparición del virus, pero cuya presencia es invocada como fundamento de distintas posiciones, y del papel que el discurso, el poder y la economía juegan en el diseño de una política en que la misma vida humana está en juego.

\section{Conclusiones}

Una conclusión que parece clara es que el concepto de evidencia ha vivido una transformación a lo largo de los siglos que la ha ido alejando paulatinamente de la idea de certeza absoluta vigente en la Antigüedad. Sin embargo, hoy nos enfrentamos a una falta de contenido preciso de este y otros términos analizados, que se 
han ido introduciendo en forma creciente en la cultura actual, con una valoración

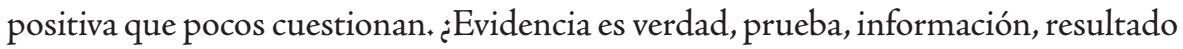
de evaluación? Quienes defienden esta posición han sacralizado la palabra, la han embellecido de objetividad, rigurosidad y utilidad, y la han contrapuesto a los mitos. Por el contrario, quienes no la comparten destacan el papel jugado en su concepción por el lenguaje, la argumentación y las creencias.

Especialmente en materia de evaluación y rediseño de políticas públicas, en el caso de varios países latinoamericanos, la revisión presentada destaca la importancia de pensar en el lenguaje empleado en su comunicación. La "evidencia" se ha vuelto un símbolo que caracteriza el lenguaje de un enfoque teórico, que se identifica con otros términos cargados de valor positivo en su discurso, como objetividad, rigurosidad, cientificidad, expertise, medición cuantitativa, modelos y datos robustos, cuasiexperimentación, estandarización, entre otros. Excepcionalmente, la "evidencia" así entendida acepta ser complementada con alguna técnica cualitativa y solo se limita a ella cuando no hay posibilidad alguna de medir en forma cuantitativa. Este modelo, fuertemente positivista, se plantea como el único serio y respetable, y se impone como argumento de autoridad donde hay otros posibles. Su enfoque no se discute en nuestra región, prácticamente no se conoce otro, a pesar que se le han señalado fallas importantes como la falta de explicación de los resultados, simplemente medidos (Banco Mundial 2004) y desde la academia existen intentos por desmitificarlo (Roth, 2009; García y Cardozo, 20I7).

Cuando efectivamente se cree con honestidad que este es el mejor enfoque, es posible debatir con pleno respeto de sus defensores, pero si se ponen en práctica procesos deliberados de manipulación del lenguaje para impedir su cuestionamiento, ya no hay solo una discrepancia teórico-metodológica, sino de fuerte contenido ético.

\section{Referencias}

Adam, Christian, Ives Steineback y Chistophe Knill

2018 "Neglected challenges to evidence-based policy-making: the problem of policy accumulation", Policy Science, 51, pp. 269-290, https://doi. org/10.1007/siro77-018-9318-4.

Aeval

2010 Fundamentos de evaluación de Políticas Públicas, Gobierno de España. 
Almeida, Alex

2017 “Cómo crear políticas públicas basadas en evidencias?", evento de apertura en la Semana de la Evidencia, Ecuador.

Bachelard, Gastón

2000 La formación del espiritu científico, ed. 23, México, Siglo xxi Editores.

Bentham, Jeremy

I843 "Rationale of Judicial Evidence", en The Works of Jeremy Bentham, vol. vi, Edimburgo, John Bowring, Simpkin, Marshall \& co.

López-Acevedo, Gladys y Michael Walton

2004 La pobreza en México: Una evaluación de las condiciones, las tendencias

Banks, Gary $y$ las estrategias del gobierno, México, Banco Mundial.

2009 Evidence-Based Policy Making: What is It? How Do We Get It?, Canberra, Australia, Anu Public Lecture Series, Productivity Commission.

Berger, Peter L. y Thomas Luckmann

I966 La construcción social de la realidad, Buenos Aires, Amorrortu.

Bertranou, Julián

2019 "El seguimiento y la evaluación de políticas públicas", Millcayac. Revista Digital de Ciencias Sociales, vi(I0), pp. I5I-187, https://doi.org/10.1590/.

Bracho, Teresa

20 "Políticas basadas en evidencia. La política pública como acción informada y objeto de investigación”, en Mauricio Merino y Guillermo Cejudo (eds.), Problemas, decisiones y soluciones, México, CIDE-FCE, pp. 29I-319.

Campbell, D. y J. Stanley

2005 Diseños experimentales y cuasiexperimentales en la investigación social, Madrid/Buenos Aires, Amorrortu.

Cardozo, Myriam

201 "Organización y métodos alternativos en la evaluación de políticas y programas sociales. La experiencia consolidada y la emergente en el caso de México", Revista Gestión y Análisis de Políticas Públicas, 5, pp. 43-59, https://doi.org/Io.24965/gapp.voi5.445.

Cardozo, Myriam

2015 Institucionalización del proceso de evaluación. Calidad y utilización de sus resultados, México, UAM-X/CESOP.

Cairney, Paul

2016 Based Policy Making, Gran Bretaña, Palgrave MacMillan. 
Cejudo, Guillermo

2010 "Discurso y políticas públicas. Enfoque constructivista", en Mauricio Merino y Guillermo Cejudo (eds.), Problemas, decisiones y soluciones, México, CIDE-FCE, pp. 93-I25.

Coe, Robert

2004 "Why kind of evidence does government need?", Evaluation and Research in Education, I8 (I-2), pp. I-II, https://doi. org/I0.1080/09500790408668305.

Coneval

2020 "Evidencia sobre la efectividad de las políticas sociales", disponible en https://www.coneval.org.mx/Evaluacion/eseps/Paginas/evidencia_efectividad_politicas_sociales.aspx. [consulta: 15/02/2020].

Coneval

2020 "Memorias del Coneval 2006-2015", disponible en https://www.coneval.org.mx/InformesPublicaciones/Documents/Memorias/Sistema-de-monitoreo-y-evaluacion-politica.pdf. [consulta: 15/02/2020].

Coneval

2020 "Identificación y clasificación del uso de la información del Coneval para la mejora de la política de desarrollo social", disponible en https://www. coneval.org.mx/quienessomos/ComoNosMedimos/Documents/ Uso_de_la_informacion_de_conEvAL_v3.pdf.[consulta: 17/02/2020].

Coneval 2020

Coneval 2020

Coneval

2020 "Incubadora de evaluaciones de impacto", disponible en https://www. coneval.org.mx/Evaluacion/ESEPs/Paginas/incubadora_impacto.aspx. [consulta: $17 / 02 / 2020$ ].

Coneval

"Memorias del Coneval 2006-2016", disponible en https://www.coneval.org.mx/InformesPublicaciones/Documents/Memorias/Memorias-Coneval-2006-2016.pdf. [consulta: 15/02/2020].

"Evaluación de la política de desarrollo social 20r8", disponible en https://www.coneval.org.mx/Evaluacion/IEPSM/IEPSM/Paginas/ IEPDS-2018.aspx. [consulta: 17/02/2020].

2020 "Información Coneval para el proceso presupuestario 2020", disponible en https://www.coneval.org.mx/Evaluacion/IEpsm/Paginas/Consideraciones2020.aspx. [consulta: 15/02/2020]. 
Confederación Patronal de la República Mexicana

2019 "Decisiones públicas basadas en evidencia. Premisa para el buen gobierno", Disponible en https://coparmex.org.mx/informacion-publica-para-un-desarrollo-con-rumbo. [consulta: 27/02/2020].

Couture, Eduardo 2005 Vocabulario jurídico, Buenos Aires, Ediciones Depalma.

Chomsky, Noam

1992 El lenguaje y el entendimiento, Barcelona, Planeta-De Agostini.

Davies, Philip

2004 "Is evidence-based government possible?", en Jerry Lee, Lectures to be presented at The 4th Annual Campbell Collaboration Colloquium, Washington.

Davies, Philip

2012 "The State of Evidence-Based Policy Evaluation and its Role in Policy Formation", National Institute Economic Review, 219, pp. 4I-52, https:// doi.org/I0.1177/002795011221900105.

De Saussure, Ferdinand

2008 Curso de lingüistica general, Buenos Aires, Losada.

Dirección de Presupuestos de Chile 2020 disponible en https://www.dipres.gob.cl. [consulta: 03/03/2020].

Droit, Roger-Pol 20II Una breve historia de la filosofía, España, Paidós.

European Evaluation Society

2020 disponible en https://www.europeanevaluation,org. [consulta: 07/032020].

Feyerabend, Paul

I975 Tratado contra el método, Madrid, Tecnos.

Fischer, Frank

2003 Reframing public policy. Discursive politics and deliberative practices, Oxford, Oxford University Pres.

Flax, Jane

I987 "Postmodernism and gender relationships in feminist theory", Signs, I2, pp. 62I-643.

Flores-Crespo, Pedro

2013 "El enfoque de la política basado en la evidencia: Análisis de su utilidad para la educación de México", Revista mexicana de investigación educativa, I8(56), pp. 265-290. 
Foucault, Michel

1966 Las palabras y las cosas. Una arqueología de las ciencias bumanas, Madrid, Siglo xxi Editores.

French, Richard

2018 "Is it time to give up on evidence-based policy? Four answers", Policy E Politics, 47(I), pp I5I-I68, DoIः I0.1332/030557318XI5333033508220.

García, Ester y Myriam Cardozo

2017 "Evaluación de impacto: más allá de la experimentación", Revista Política

Gergen, Kenneth $y$ Cultura, 47, pp. 65-9I.

2007 Construccionismo social. Aportes para el debate y la práctica, Bogotá, Uniandes-Ceso.

Giddens, Anthony

2006 Sociology, Cambridge, Polity Press.

Head, Brian

2010 "Reconsidering evidence-based policy: Key issues and challenges", Journal Policy and Society, Vol. 29: 2, pp. 77-94, https://doi.org/ı0.10I6/j. polsoc.2010.03.00I.

Hernández, Gonzalo y Ricardo Mujica

2008 "Avances y retos de políticas de desarrollo social en México 1996-2006", en Teresa González de Cossío, Juan Rivera Dommarco, Gladys López Acevedo y Gloria M. Rubio Soto (eds.), Nutrición y Pobreza. Política Pública basada en Evidencia, México, Banco Mundial y Sedesol, pp. 23-33.

Hernández, Gonzalo y Ricardo Mujica

2009 "Mejorando el desempeño del presupuesto: Políticas públicas de nutrición basadas en la evidencia", Nota 8, disponible en https://www.coneval.org.mx/rw/resource/coneval/med_pobreza/Banco_mundial/ Nota8.pdf, basada en el resumen ejecutivo de la publicación anterior. [consulta: $1 \mathrm{I} / 03 / 2020$ ].

Huerta Rosales, Moisés

2018 "Evaluación basada en evidencias, un nuevo enfoque de evaluación por competencias", Revista de Investigación Universitaria Le Cordon Bleu 5(I), pp. 159-I7I, https://doi.org/IO.36955/RIULCB.2018v5nI.00II. 
Jaramillo, Juan, Diana López y Felipe Castro

2018 Evaluación de impacto del Programa de Incentivos Docentes, https://colaboracion.dnp.gov.co/CDT/Sinergia/Documentos/Evaluacion_incenti-

Lefebvre, Henri vos_docentes_Documento_vf.pdf. [consulta: 17/02/2020].

1981 Lógica formal, lógica dialéctica, México, Siglo xxi Editores.

Le Moigne, Jean-Louis

I994 Le constructivisme, t. I, París, ESF.

Leuz, Christian

2018 "Evidence-based policy making: promise, challenges and opportunities for accounting and financial markets research", Accounting and Business Research, 48(5), pp. 582-608, https://doi.org/I0.1080/o00I4788.2018.I 470151.

Lyotard, Jean François

1984 The postmodern condition: A report on knowledge, Minneapolis, University of Minnesota Press.

Majone, Giandomenico

1992 Evidencia, argumentación y persuasión en la formulación de politicas, México, Colegio Nacional de Ciencias Políticas y Administración Pública/Fondo de Cultura Económica.

Majone, Giandomenico

1997 "Los usos del análisis de políticas", en Luis F. Aguilar (ed.), La hechura de las Políticas, México, M. A. Porrúa, pp. 34I-365.

Marías, Julián

1980 Historia de la filosofía, ed. 32, Madrid, Biblioteca de la Revista de Occidente.

McNamee, Sheila y Dian Marie Hosking

2012 Research and social change. A relational constructionist approach, Nueva York, Routledge.

Ministerio de las Administraciones Públicas

2001 Modernizar la administración. Libro Blanco del Gobierno Británico 1999,

Documento 23, España, INAP.

Montes de Oca, Francisco

1984 Lógica, México, Porrúa.

Morin, Edgar

1998 Introducción al pensamiento complejo, Barcelona, Gedisa. 
Olivé, León

I988 Racionalidad, México, Siglo xxi Editores/UnAm.

Parkhurst, Justin

2017 The politics of evidence: from evidence-based policy to de good governance of evidence, Routledge, Reino Unido, Routledge Studies in Governance and Public Policy.

Pawson, Ray

2006 Evidence-based policy: A realistic perspective, Londres, Sage Publications. Pérez Yarahuán, Gabriela y Claudia Maldonado Trujillo (eds.)

2015 Panorama de los sistemas nacionales de monitoreo y evaluación en América Latina, México, CIDE/Clear LAC.

Pinilla, Rafael

2006 "Política basada en la evidencia para la renovación del Edo de Bienestar", XIII Encuentro de Economía Pública, 2 y 3 de febrero, Hotel Playadulce, Almería, España.

Popper, Karl 2007 Los dos problemas fundamentales de la epistemología, España, Tecnos.

Ramírez, María Clemencia

2010 Presentación de "La antropología de la política pública", Antípoda. Revista de Antropología y Arqueología, Io, pp. 13-17, https;//doi.org/10.7440/ antipodaio.2010.02.

Raya Díez, Esther y Joseba Zalakain

201 "Políticas por evidencias: la información en los procesos de toma de decisiones en los proyectos sociales”, en Esther Raya (coord.), Herramientas para el diseño de proyectos sociales, España, Universidad de La Rioja, pp. 25-35.

Reygadas, Pedro 2009 Argumentación y discurso, México, El Colegio de San Luis.

Reygadas, Pedro y Juan M. Contreras

2009 La protofilosofía griega (siglos viI a V A.C.): entre el Mediterráneo, el Egeo y el Jónico. Mapa de la bistoria mundial de las filosofías, México, El Colegio de San Luis.

Roth, Arnold

2009 "La evaluación de políticas públicas en Colombia: una mirada crítica a partir de las prácticas evaluativas oficiales de los programas de la Red de Apoyo Social", Revista del CLAD Reforma y Democracia, 45, pp. I6I-I86. 
Segura Fernández, Karen

2012 Políticas nacionales basadas en evidencia: significado e implicaciones, OEA/ Ser.L/XIV.2.5I, CICAD/doc.1945/I2.

Shore, Cris

2010 "La antropología y el estudio de la política pública. Reflexiones sobre la 'formulación' de las políticas", Antípoda. Revista de Antropología y Arqueología, IO, pp. 21-49, https://doi.org/10.7440/antipodaIo.2010.03. Simons, Helen

2004 "Utilizing evaluation evidence to enhance professional practice", Evaluation, IO (4), pp. 410-429, DOI* I0.1177/1356389004050284.

Sinergia

2020 https://sinergia.dnp.gov.co. [consulta: 20/03/2020].

Torres, Rafael

2015 "Políticas basadas en la evidencia. La paradoja de la evidencia científica", Programa Ciencia, Tecnología e Innovación, Chile, Biblioteca del Congreso Nacional.

Xirau, Ramón

2011 Introducción a la bistoria de la filosofía, México, unam.

Weiss, Carol

1979 "The many meanings of research utilization", Public Administration Review 39 (5), pp. 426-43I, DOI: 10.2307/3109916.

Myriam Irma Cardozo Brum

Es doctora en Ciencias Políticas y Sociales por la Universidad Nacional Autónoma de México y cuenta con reconocimiento del Sistema Nacional de Investigadores (Conacyt), nivel Ir. De 1992 a 1994 ocupó un Cátedra de Excelencia Académica en el Departamento de Política y Cultura de la Universidad Autónoma Metropolitana-Xochimilco (UAM-X, México), posteriormente ganó una plaza definitiva por concurso de oposición. Ha fungido como coordinadora de la Licenciatura en Política y Gestión Social (1998-200I), coordinadora de la Maestría en Políticas Públicas (2002-2003) y jefa de Área de Política y Gestión Pública (2007-2009). Es docente a nivel de Licenciatura, Maestría y Doctorado, impartiendo fundamentalmente el módulo de Evaluación de la Maestría en Políticas Públicas, Evaluación de Proyectos Sociales en la Licenciatura en Política y Gestión Social, cursos de metodología y seminarios de tesis. 
Desde 2002 ha desarrollado un proyecto de investigación sobre evaluación de políticas y programas públicos que le permitió, entre otras actividades, integrar el Consejo de Evaluación del Desarrollo Social del Distrito Federal (ahora Evalúa CDMX) en 2008-2012 y nuevamente a partir de 20I9, realizar tres estancias de investigación en Cachan (Francia), Montréal (Canadá) y Toulouse (Francia). Ha presentado trabajos de investigación en este tema en 123 eventos, publicado 42 artículos y capítulos de libro y ir libros. Recientemente coordinó el Congreso Internacional de Evaluación de Políticas y Programas Públicos.

Citar como: Myriam Irma Cardozo Brum (202I), “Evidencia: conceptos y usos en la evaluación de políticas y programas públicos", Iztapalapa. Revista de Ciencias Sociales y Humanidades, núm. 90, año 42, enero-junio de 202I, ISSN: 2007-9176; pp. 205-232. Disponible en <http://revistaiztapalapa.izt. uam.mx/index.php/izt/issue/archive $>$. 